Pacific

Journal of

Mathematics

\title{
SPANNING TREES AND RANDOM WALKS ON WEIGHTED GRAPHS
}

Xiao Chang, Hao Xu and Shing-Tung Yau

Volume $273 \quad$ No. 1

January 2015 


\title{
SPANNING TREES AND RANDOM WALKS ON WEIGHTED GRAPHS
}

\author{
Xiao Chang, Hao Xu and Shing-Tung Yau
}

\begin{abstract}
Using two graph invariants arising from Chung and Yau's discrete Green's function, we derive explicit formulas and new estimates of hitting times of random walks on weighted graphs through the enumeration of spanning trees.
\end{abstract}

\section{Introduction}

Every reversible Markov chain can be viewed as a random walk on a weighted undirected graph $G=(V, E)$ with edge weights $w_{x y}$. We may assume that $G$ has no multiedges but may have a loop of weight $w_{x x}$ at each vertex $x$. The weighted degree $d_{x}$ of $x$ is the sum of all $w_{x y}, y \in V$. The volume of a graph is $\operatorname{vol}(G)=\sum_{v \in V} d_{v}$.

The Laplacian of $G$ is the matrix $L=D-A$, where $D$ is the diagonal matrix whose entries are $d_{x}, x \in V$ and $A$ is the adjacency matrix of $G$. Chung's normalized Laplacian, $\mathscr{L}=D^{-1 / 2} L D^{-1 / 2}$, is

$$
\mathscr{L}(x, y)= \begin{cases}1-w_{x x} / d_{x} & \text { if } x=y, \\ -w_{x y} / \sqrt{d_{x} d_{y}} & \text { if } x \sim y, \\ 0 & \text { otherwise }\end{cases}
$$

where $x \sim y$ denotes that $x$ and $y$ are adjacent. Here we assume that $d_{x} \neq 0$ for all $x \in V$, since for a random walk it is natural to impose that $G$ is connected and $w_{x y}>0$ for all $x y \in E$.

If $G$ is connected, denote by $0=\lambda_{0}<\lambda_{1} \leq \lambda_{2} \leq \cdots \leq \lambda_{n-1}$ the eigenvalues of $\mathscr{L}$ with the corresponding orthonormal basis of eigenvectors $\phi_{0}, \phi_{1}, \ldots, \phi_{n-1}$, as $n \times 1$ column vectors. Obviously $\phi_{0}(x)=\sqrt{d_{x} / \operatorname{vol}(G)}$.

Chung and Yau [2000] defined the discrete Green's function $\mathscr{G}$ by

$$
\mathscr{G}=\sum_{j=1}^{n-1} \frac{1}{\lambda_{j}} \phi_{j} \phi_{j}^{*}
$$

MSC2010: 05C81.

Keywords: hitting time, random walk, spanning tree. 
which is uniquely determined by the relations $\mathscr{G} \mathscr{L}=\mathscr{L} \mathscr{G}=I-P_{0}$ and $\mathscr{G} P_{0}=0$, where $P_{0}=\phi_{0} \phi_{0}^{t}$ is an $n \times n$ matrix.

A random walk on $G$ is a Markov chain on $V$ with transition probability matrix $\left(p_{x y}\right)_{x, y \in V}$, where

$$
p_{x y}= \begin{cases}w_{x y} / d_{x} & \text { if } x \sim y \\ 0 & \text { otherwise }\end{cases}
$$

The hitting time $H(x, y)$ is the expected number of steps to reach vertex $y$ when started from vertex $x$. By using a result in [Aldous and Fill 2014], Chung and Yau proved an expression for $H(x, y)$ in terms of the discrete Green's function:

Theorem 1.1 [Chung and Yau 2000]. On a connected graph $G$, the hitting time $H(x, y)$ and Green's function $\mathscr{G}(x, y)$ satisfy

$$
H(x, y)=\operatorname{vol}(G)\left(\frac{\mathscr{G}(y, y)}{d_{y}}-\frac{\mathscr{G}(x, y)}{\sqrt{d_{x} d_{y}}}\right) .
$$

For a weighted graph $G$, we denote by $\Omega(G)$ the set of spanning trees of $G$. For $T \in \Omega(G)$, define the weight $w(T)$ of $T$ to be $\prod_{e \in T} w_{e}$. Let $\tau(G)$ be the weighted counting of spanning trees:

$$
\tau(G)=\sum_{T \in \Omega(G)} w(T)
$$

Below is a typical identity expressing hitting times in terms of spanning trees (see Theorem 2.11) arising from our study of Chung and Yau's discrete Green's function.

Theorem 1.2. Let $G$ be a connected weighted graph and $x, y \in V(G)$. Then

$$
H(x, y)=\frac{1}{\tau(G)} \sum_{u \in V(G)} d_{u} \sum_{\substack{P \in \mathscr{P}_{G}(x, u) \\ y \notin P}} \prod_{e \in E(P)} w_{e} \tau(G /\{P, y\}) .
$$

The paper is organized as follows. In Section 2, we introduce two graph invariants and use them to derive explicit formulas for Chung and Yau's discrete Green's functions and hitting times of random walks on weighted graphs. In Section 3, we apply our formulas to obtain various estimates of hitting times on weighted graphs. In Section 4, we prove an explicit formula for hitting times of random walks on infinite trees. In Section 5, we apply our work to improve estimates of hitting times under different weight schemes on a given simple finite graph.

\section{The hitting time of random walks on weighted graphs}

Kirchhoff discovered his matrix-tree theorem in 1847 in his work on electrical networks, and this theorem gives an efficient way to calculate $\tau(G)$ using linear algebra. 
Theorem 2.1 (Kirchhoff's matrix-tree theorem). Let $G$ be a connected weighted graph.

(i) If the Laplacian $L$ of $G$ has eigenvalues $0=\mu_{0}<\mu_{1} \leq \cdots \leq \mu_{n-1}$, then

$$
\prod_{k=1}^{n-1} \mu_{k}=n \tau(G)
$$

(ii) Let $L_{i j}$ be the matrix obtained from $L$ by deleting the $i$-th row and $j$-th column. Then all cofactors $(-1)^{i+j} \operatorname{det}\left(L_{i j}\right)$ of $L$ are equal and

$$
\operatorname{det} L_{i i}=n \tau(G) \quad \text { for all } 1 \leq i \leq n \text {. }
$$

We also need the following version of Kirchhoff's matrix-tree theorem for weighted graphs. A proof, with slight changes, can be found in the cited reference.

Theorem 2.2 [Chung 2011, Theorem 1]. For a connected weighted graph $G=$ $(V, E)$, we have

$$
\prod_{k=1}^{n-1} \lambda_{k}=\frac{\operatorname{vol}(G) \tau(G)}{\prod_{v \in V} d_{v}}
$$

where $0=\lambda_{0}<\lambda_{1} \leq \cdots \leq \lambda_{n-1}$ are eigenvalues of $\mathscr{L}$ and $\tau(G)$ is defined in (2).

A weighted graph is a graph $G$ equipped with a function $w: E(G) \rightarrow \mathbb{R}_{+}$that assigns a positive number to each edge. In the following, we fix a weighted graph $(G, w)$ and introduce two invariants for any induced subgraph $S$ of $G$. Consider the matrix

$$
B(x, y)= \begin{cases}d_{x}^{2} s+d_{x}-w_{x x} & \text { if } x=y \\ d_{x} d_{y} s-w_{x y} & \text { if } x \sim y \\ d_{x} d_{y} s & \text { otherwise }\end{cases}
$$

where $d_{x}=w_{x x}+\sum_{y \sim x} w_{x y}$. Denote by $B_{S}$ the principle submatrix of $B$ on indices corresponding to the vertices of $S$; we define $R(S)$ and $Z(S)$ by

$$
\operatorname{det} B_{S}=R(S)+Z(S) \cdot s \text {. }
$$

In particular, $R(\varnothing)=1, Z(\varnothing)=0$ for the empty subgraph $\varnothing$, and $R(\{x\})=d_{x}-w_{x x}$, $Z(\{x\})=d_{x}^{2}$ for any $x \in V(G)$. 
In the following four lemmas, $S$ is an arbitrary induced subgraph of a fixed weighted graph $(G, w)$. The proofs of these lemmas are similar to those in $[\mathrm{Xu}$ and Yau 2013a, Section 2], where we considered unweighted simple graphs with $w_{e} \equiv 1$ for all $e \in E(G)$.

Lemma 2.3. If $S$ has $k$ connected components $S_{1}, \ldots, S_{k}$, then

$$
R(S)=\prod_{i=1}^{k} R\left(S_{i}\right), \quad Z(S)=\sum_{i=1}^{k} Z\left(S_{i}\right) \prod_{\substack{j=1 \\ j \neq i}}^{k} R\left(S_{j}\right) .
$$

Lemma 2.4. For any fixed vertex $x \in V(S)$, we have

$$
R(S)=\left(d_{x}-w_{x x}\right) R(S-\{x\})-\sum_{\substack{y \in V(S) \\ y \sim x}} w_{x y} \sum_{P \in \mathscr{P}_{S}(x, y)} \prod_{e \in E(P)} w_{e} R(S-\{P\}),
$$

and

$$
\begin{gathered}
Z(S)=\left(d_{x}-w_{x x}\right) Z(S-\{x\}) \\
-\sum_{\substack{y \in V(S) \\
y \sim x}} w_{x y} \sum_{P \in \mathscr{P}_{S}(x, y)} \prod_{e \in E(P)} w_{e} Z(S-\{P\})+\left(d_{x}-w_{x x}\right)^{2} R(S-\{x\}) \\
+\sum_{\substack{u, v \in V(S) \\
u \neq v}} d_{u} d_{v} \sum_{\substack{P_{1} \in \mathscr{P}_{S}(x, u) \\
P_{2} \in \mathscr{P}_{S}(x, v) \\
P_{1} \cap P_{2}=x}} \prod_{e \in E\left(P_{1} \cup P_{2}\right)} w_{e} R\left(S-\left\{P_{1}, P_{2}\right\}\right),
\end{gathered}
$$

where $\mathscr{P}_{S}(x, y)$ is the set of all simple paths (with no repeated vertices) connecting $x$ and $y$ in $S$. We assume that $\mathscr{P}_{S}(x, x)$ consists of the trivial path $\{x\}$ only. Here $S-\{P\}$ means the graph obtained by removing $P$ together with incident edges.

Lemma 2.5. We have

$$
Z(S)=\sum_{x, y \in V(S)} d_{x} d_{y} \sum_{P \in \mathscr{P}_{S}(x, y)} \prod_{e \in E(P)} w_{e} R(S-\{P\}) .
$$

Lemma 2.6. Regarding $G$ as a subgraph of itself, we have $R(G)=0$ and $Z(G)=$ $\operatorname{vol}(G)^{2} \tau(G)$. For any $x, y \in V(G)$, we have

$$
R(G-\{x\})=\sum_{P \in \mathscr{P}_{G}(x, y)} \prod_{e \in E(P)} w_{e} R(G-\{P\})=\tau(G) .
$$

Now we come to an explicit formula for the Green's function expressed in terms of the above two invariants. 
Theorem 2.7. For a connected graph $G$ and $x, y \in V(G)$, the value of the Green's function $\mathscr{G}(x, y)$ is equal to

$$
\begin{aligned}
\frac{\sqrt{d_{x} d_{y}}}{\operatorname{vol}(G)^{2} \tau(G)}\left(\sum_{P \in \mathscr{P}_{G}(x, y)} \prod_{e \in E(P)} w_{e}(R(G-\{P\})+Z(G-\{P\}))\right. & \\
& \left.-\sum_{\substack{u, v \in V(G) \\
u \neq v}} d_{u} d_{v} \sum_{\substack{P_{1} \in \mathscr{P}_{G}(x, u) \\
P_{2} \in \mathscr{P}_{G}(y, v) \\
P_{1} \cap P_{2}=\varnothing}} \prod_{e \in E\left(P_{1} \cup P_{2}\right)} w_{e} R\left(G-\left\{P_{1}, P_{2}\right\}\right)\right) \\
& -\frac{\sqrt{d_{x} d_{y}}}{\operatorname{vol}(G)^{2}} .
\end{aligned}
$$

In particular, when $x=y$,

$$
\mathscr{G}(y, y)=\frac{d_{y}}{\operatorname{vol}(G)^{2} \tau(G)}(R(G-\{y\})+Z(G-\{y\}))-\frac{d_{y}}{\operatorname{vol}(G)^{2}} .
$$

Proof. The proof is almost the same as that of [Xu and Yau 2013a, Theorem 2.9].

Theorem 2.8. Given a connected graph $G$ and $x, y \in V(G)$, the expected hitting time $H(x, y)$ satisfies

$$
\begin{gathered}
H(x, y)=\frac{1}{\operatorname{vol}(G) \tau(G)}\left(Z(G-\{y\})-\sum_{P \in \mathscr{P}_{G}(x, y)} \prod_{e \in E(P)} w_{e} Z(G-\{P\})\right. \\
\left.+\sum_{\substack{u, v \in V(G) \\
u \neq v}} d_{u} d_{v} \sum_{\substack{P_{1} \in \mathscr{P}_{G}(x, u) \\
P_{2} \in \mathscr{P}_{G}(y, v) \\
P_{1} \cap P_{2}=\varnothing}} \prod_{e \in E\left(P_{1} \cup P_{2}\right)} w_{e} R\left(G-\left\{P_{1}, P_{2}\right\}\right)\right) .
\end{gathered}
$$

Proof. This follows from Theorems 1.1 and 2.7 and Lemma 2.6.

Corollary 2.9. On a connected weighted graph $G, H(x, y)=H(y, x)$ for any $x, y \in V(G)$ if and only if $Z(G-\{x\})$ is independent of $x \in V(G)$.

Proof. By (10), we have

$$
H(x, y)-H(y, x)=\frac{1}{\operatorname{vol}(G) \tau(G)}(Z(G-\{y\})-Z(G-\{x\})),
$$

which implies the corollary.

Corollary 2.10. On a connected weighted graph $G, H(x, y)=H(y, x)$ for any $x, y \in V(G)$ if and only if $\left.\operatorname{det} B_{G-\{x\}}\right|_{s=1}$ is independent of $x \in V(G)$.

Proof. From det $\left.B_{G-\{x\}}\right|_{s=1}=R(G-\{x\})+Z(G-\{x\})$, the conclusion follows from Lemma 2.6 and the previous corollary. 
Theorem 2.11. Let $G$ be a connected weighted graph and $x, y \in V(G)$. Then

$$
H(x, y)=\frac{1}{\tau(G)} \sum_{u \in V(G)} d_{u} \sum_{\substack{P \in \mathscr{P}_{G}(x, u) \\ y \notin P}} \prod_{e \in E(P)} w_{e} R(G-\{P, y\}) .
$$

In fact, $R(G-\{P, y\})=\tau(G /\{P, y\})$, where $G /\{P, y\}$ is obtained from $G$ by contracting $\{P, y\}$ to a point.

Proof. The proof is almost identical to that of [Xu and Yau 2013b, Theorem 2.7]. $R(G-\{P, y\})=\tau(G /\{P, y\})$ follows from Theorem 2.1(ii).

Corollary 2.12. For any connected weighted graph $G$, we have

$$
Z(G-\{x\})=\tau(G) \sum_{y \in V(G)} d_{y} H(y, x) .
$$

Proof. By (8) and (11), we have

$$
\begin{aligned}
Z\left(G-\{x\}, d_{G}\right) & =\sum_{u, y \in V(G-\{x\})} d_{u} d_{y} \sum_{P \in \mathscr{P}_{G-\{x\}}(u, y)} \prod_{e \in E(P)} w_{e} R(G-\{P, x\}) \\
& =\sum_{y, u \in V(G)} d_{y} d_{u} \sum_{\substack{P \in \mathscr{P}_{G}(y, u) \\
x \notin P}} \prod_{e \in E(P)} w_{e} R(G-\{P, x\}) \\
& =\tau(G) \sum_{y \in V(G)} d_{y} H(y, x) .
\end{aligned}
$$

\section{Identities and estimates of hitting times}

It is natural to regard a weighted graph as an electrical network, where an edge $x y$ has conductance $w_{x y}$ and hence resistance $1 / w_{x y}$. Chandra et al. [1989] proved that the commute time $\kappa(x, y):=H(x, y)+H(y, x)$ can be expressed in terms of the effective resistance $R_{x y}$ between $x, y$,

$$
\kappa(x, y)=\operatorname{vol}(G) R_{x y} .
$$

The effective resistance $R_{x y}$ can be expressed in terms of spanning trees (cf. Theorem 3.2):

$$
R_{x y}=\frac{\tau(G /\{x, y\})}{\tau(G)} .
$$

Tetali's formula [1991] expresses $H(x, y)$ in terms of effective resistances:

$$
H(x, y)=\frac{1}{2} \sum_{z \in V(G)} d_{z}\left(R_{x y}+R_{y z}-R_{x z}\right) .
$$

We have the following well-known upper bound of $R_{x y}$. 
Theorem 3.1. Given a connected graph $G$ and $x, y \in V(G)$, we have $R_{x y} \leq d(x, y)$, where the distance $d(x, y)$ between $x, y$ is defined by

$$
d(x, y)=\min \left\{\sum_{e \in E(P)} \frac{1}{w_{e}} \mid P \in \mathscr{P}_{G}(x, y)\right\} .
$$

As remarked in [Lovász 1996, Corollary 4.2], the following formula could be proved by the method of electric networks. Here we give a proof by using (11).

Theorem 3.2. Let $x, y \in V(G)$ be two distinct vertices of a connected weighted graph $G$. Then we have

$$
H(x, y)+H(y, x)=\operatorname{vol}(G) \frac{\tau(G /\{x, y\})}{\tau(G)},
$$

where $G /\{x, y\}$ is obtained from $G$ by contracting $\{x, y\}$ to a point.

Proof. Define a graph $G^{\prime}$ by

$$
G^{\prime}= \begin{cases}G & \text { if } x \sim y, \\ G \cup\{x y\} & \text { otherwise. }\end{cases}
$$

Namely, we modify $G$ by adding an edge $x y$ if $x, y$ are not adjacent.

If $u \neq x, y$, define

$\Omega_{1}=\left\{T \in \Omega\left(G^{\prime}\right) \mid T\right.$ contains $x y$ and a path from $u$ to $x$ not containing $\left.y\right\}$,

$\Omega_{2}=\left\{T \in \Omega\left(G^{\prime}\right) \mid T\right.$ contains $x y$ and a path from $u$ to $y$ not containing $\left.x\right\}$,

$\Omega_{3}=\left\{T \in \Omega\left(G^{\prime}\right) \mid T\right.$ contains $\left.x y\right\}$.

It is not difficult to see that $\Omega_{1} \cup \Omega_{2}=\Omega_{3}=\Omega(G /\{x, y\})$. (More precisely, $\Omega_{3}$ is in one-to-one correspondence with $\Omega(G /\{x, y\})$.) Then, by (11),

$$
H(x, y)+H(y, x)
$$

$$
\begin{aligned}
& =\frac{1}{\tau(G)} \sum_{u \in V(G)} d_{u}\left(\sum_{\substack{P \in \mathscr{P}_{G}(x, u) \\
y \notin P}} \prod_{e \in E(P)} w_{e} \tau(G /\{P, y\})\right. \\
& \left.+\sum_{\substack{P \in \mathscr{P}_{G}(y, u) \\
x \notin P}} \prod_{e \in E(P)} w_{e} \tau(G /\{P, x\})\right) \\
& =\frac{1}{\tau(G)} \sum_{u \in V(G)} d_{u}\left(\sum_{T \in \Omega_{1}} \prod_{e \in T} w_{e}+\sum_{T \in \Omega_{2}} \prod_{e \in T} w_{e}\right) \\
& =\frac{1}{\tau(G)} \sum_{u \in V(G)} d_{u} \tau(G /\{x, y\}) .
\end{aligned}
$$

The term in parenthesis on the third line is equal to $\tau(G /\{x, y\})$, independently of $u \in V(G)$. 
In the rest of this section, we apply Theorem 2.11 to prove some estimates for the hitting time on weighted graphs. It is interesting to see the role of edge weights in these estimates.

Corollary 3.3. Let $G$ be a connected weighted graph with $n$ vertices and $x, y \in$ $V(G)$. Then

$$
H(x, y) \leq(n-1)^{2} \frac{d_{\max }}{w_{\min }},
$$

where $d_{\max }=\max \left\{d_{v} \mid v \in V(G)\right\}$ and $w_{\min }=\min \left\{w_{e} \mid e \in E(G)\right\}$.

Proof. Fix $x, y, u \in V(G)$ with $y \neq u$. Given a spanning tree $T \in \Omega(G)$ and an edge $e \in E(T)$, denote by $T(e)$ the subgraph of $G^{\prime}$ obtained from $T$ by removing $e$ and adding an edge $u y$ if $u y \notin E(T)$, namely,

$$
T(e)= \begin{cases}T & \text { if } u y \in T, \\ T \cup\{u y\}-\{e\} & \text { if } u y \notin T .\end{cases}
$$

Define a subset $S$ of $\Omega(G) \times E(G)$ by

$$
S=\left\{(T, e) \mid T \in \Omega(G), e \in E(T), T(e) \in \Omega\left(G^{\prime}\right)\right\}
$$

and let $S^{\prime}=\left\{T \in \Omega\left(G^{\prime}\right) \mid T\right.$ contains $\left.u y\right\}$. Then the map $(T, e) \rightarrow T(e)$ is a surjective map from $S$ to $S^{\prime}$. Since

$$
\begin{aligned}
& \bigcup_{\substack{P \in \mathscr{P}_{G}(x, u) \\
y \notin P}} \Omega(G /\{P, y\}) \\
& \quad=\left\{T \in \Omega\left(G^{\prime}\right) \mid T \text { contains } u y \text { and a path from } u \text { to } x \text { not passing through } y\right\}
\end{aligned}
$$
is a subset of $S^{\prime}$ and the left-hand side is a disjoint union over $P \in \mathscr{P}_{G}(x, u), y \notin P$, we have

$$
\sum_{\substack{P \in \mathscr{P}_{G}(x, u) \\ y \notin P}} \prod_{e \in E(P)} w_{e} \tau(G /\{P, y\}) \leq(n-1) \tau(G) \frac{1}{w_{\min }} .
$$

Then (17) follows from (11).

Corollary 3.4. Let $G$ be a connected weighted graph and $x y \in E(G)$. Then

$$
H(x, y) \leq \frac{\operatorname{vol}(G)-d_{y}}{w_{x y}} .
$$

Proof. Fix $x, y, u \in V(G)$ with $y \neq u$. It is not difficult to see that

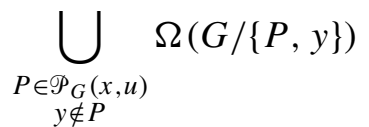

$=\{T \in \Omega(G) \mid T$ contains $x y$ and a path from $u$ to $x$ not passing through $y\}$ 
is a subset of $\Omega(G)$ and the left-hand side is a disjoint union over $P \in \mathscr{P}_{G}(x, u)$, $y \notin P$. Thus (11) implies that

$$
H(x, y) \leq \frac{1}{\tau(G)} \sum_{\substack{u \in V(G) \\ u \neq y}} d_{u} \frac{\tau(G)}{w_{x y}}=\frac{\operatorname{vol}(G)-d_{y}}{w_{x y}},
$$

as claimed.

Corollary 3.5. Let $G$ be a connected weighted graph. Then for any two distinct vertices $x, y \in V(G)$, we have

$$
H(x, y) \leq \max \left\{\frac{d_{u}}{w_{y u}} \mid u \in \mathscr{S}\right\},
$$

where $\mathscr{S}=\{u \in V(G) \mid$ there is a path from $x$ to $u$ not passing through $y\}$.

Proof. Fix two distinct vertices $x, y \in V(G)$. We may assume that $w_{y u}>0$ for all $u \in \mathscr{Y}$, i.e., there is an edge connecting $y$ and $u$. Otherwise the right-hand side of (18) is infinite. Define $\Omega_{x y}=\{T \in \Omega(G) \mid x y \in T\}$ and

$V_{T}=\{u \in V(G) \mid T$ contains a path from $x$ to $u$ not passing through $y\}$.

Let $S=\left\{(T, u) \mid T \in \Omega_{x y}, u \in V_{T}\right\}$. Define a map $f: S \rightarrow \Omega(G)$ by

$$
f(T, u)= \begin{cases}T & \text { if } u=x, \\ \{T-x y\} \cup\{u y\} & \text { if } u \neq x,\end{cases}
$$

where we used the fact that $d_{y}=n-1$. It is not difficult to see that $f$ is injective. Thus, we have

$$
\begin{aligned}
\sum_{u \in V(G)} d_{u} \sum_{\substack{P \in \mathscr{P}_{G}(x, u) \\
y \notin P}} \prod_{e \in E(P)} w_{e} \tau(G /\{P, y\}) & =\sum_{u \in V(G)} \frac{d_{u}}{w_{y u}} \sum_{(T, u) \in S} w(f(T, u)) \\
& \leq \max \left\{\frac{d_{u}}{w_{y u}} \mid u \in \mathscr{S}\right\} \tau(G) .
\end{aligned}
$$

Therefore (11) implies (19).

There is a direct probabilistic proof of Corollary 3.5 (see [Xu and Yau 2013b, Remark 2.14]). In fact, all the above three corollaries may be obtained from the following more refined estimates (see also [Chang and $\mathrm{Xu} \geq 2015$ ]):

Theorem 3.6. Let $G$ be a connected weighted graph. Then

$$
H(x, y) \leq \max \left\{d_{u} \mid u \in \Gamma(y), u \neq y\right\}+\sum_{\substack{u \in V(G), u \neq y \\ u \notin \Gamma(y)}} d_{u} \min \{d(x, y), d(u, y)\},
$$

where $\Gamma(y)$ is the set of vertices adjacent to $y$ and $d(x, y)$ is defined in Theorem 3.1. 
Proof. We split the right-hand side of (11) into two terms:

$$
\begin{aligned}
H(x, y)=\frac{1}{\tau(G)} \sum_{u \in \Gamma(y)} & d_{u} \sum_{\substack{P \in \mathscr{P}_{G}(x, u) \\
y \notin P}} \prod_{e \in E(P)} w_{e} \tau(G /\{P, y\}) \\
& +\frac{1}{\tau(G)} \sum_{\substack{u \in V(G) \\
u \notin \Gamma(y)}} d_{u} \sum_{\substack{P \in \mathscr{P}_{G}(x, u) \\
y \notin P}} \prod_{\substack{e \in E(P) \\
w_{e}}} w_{e}(G /\{P, y\}) .
\end{aligned}
$$

If $u \in \Gamma(y)$, then define

$\Omega_{u y}=\{T \in \Omega(G) \mid T$ contains $u y$ and a path from $x$ to $u$ not passing through $y\}$.

Since $\bigcup_{u \in \Gamma(y)} \Omega_{u y}$ is a disjoint union in $\Omega(G)$ and

$$
\sum_{\substack{P \in \mathscr{P}_{G}(x, u) \\ y \notin P}} \prod_{e \in E(P)} w_{e} \tau(G /\{P, y\})=\sum_{T \in \Omega_{u y}} w(T) \quad \text { for all } u \in \Gamma(G),
$$

the first summand in the right-hand side of (20) satisfies

(21) $\frac{1}{\tau(G)} \sum_{u \in \Gamma(y)} d_{u} \sum_{\substack{P \in \mathscr{P}_{G}(x, u) \\ y \notin P}} \prod_{e \in E(P)} w_{e} \tau(G /\{P, y\}) \leq \max \left\{d_{u} \mid u \in \Gamma(y), u \neq y\right\}$.

By using (14) and the inequality

$$
\sum_{\substack{P \in \mathscr{P}_{G}(x, u) \\ y \notin P}} \prod_{e \in E(P)} w_{e} \tau(G /\{P, y\}) \leq \min \{\tau(G /\{x, y\}), \tau(G /\{u, y\})\},
$$

the second summand in the right-hand of (20) satisfies

$$
\begin{aligned}
& \frac{1}{\tau(G)} \sum_{\substack{u \in V(G) \\
u \notin \Gamma(y)}} d_{u} \sum_{\substack{P \in \mathscr{P}_{G}(x, u) \\
y \notin P}} \prod_{e \in E(P)} w_{e} \tau(G /\{P, y\}) \\
& \leq \sum_{\substack{u \in V(G) \\
u \notin \Gamma(y)}} d_{u} \min \left\{R_{x y}, R_{u y}\right\} \leq \sum_{\substack{u \in V(G) \\
u \notin \Gamma(y)}} d_{u} \min \{d(x, y), d(u, y)\} .
\end{aligned}
$$

The last inequality follows from Theorem 3.1. So (19) follows from (21) and (23).

Corollary 3.7. Let $G$ be a connected weighted graph. Then

$$
H(x, y) \leq \sum_{\substack{u \in V(G) \\ u \neq y}} d_{u} \min \left\{R_{x y}, R_{u y}\right\} \leq \sum_{\substack{u \in V(G) \\ u \neq y}} d_{u} \min \{d(x, y), d(u, y)\} .
$$

Proof. The first inequality follows from (14), (22) and Theorem 2.11. The second inequality follows from Theorem 3.1. 


\section{Some examples}

First we consider infinite but locally finite connected graphs. Since an infinite (locally finite) graph can be considered as a limit of a sequence of finite graphs, the hitting time formula (11) is still valid as long as the limit exists. A weighted tree $T$ is a locally finite tree (possibly with loops) whose edges are assigned positive weights.

Theorem 4.1. Let $x, y$ be two distinct vertices of a weighted tree $T$, and denote by $P_{x y}$ the path $\left[x=v_{0}, v_{1}, \ldots, v_{k-1}, v_{k}=y\right]$ connecting $x$ to $y$. For any $v_{i} \in V\left(P_{x y}\right)$, we denote by $T_{i}$ the component of $T-E\left(P_{x y}\right)$ that contains $v_{i}$, and denote by $w_{i-1, i}$ the weight of the edge $v_{i-1} v_{i}$. Then the hitting time $H(x, y)$ is given by

$$
H(x, y)=\sum_{j=0}^{k-1}\left(\sum_{u \in T_{j}} d_{u}\right)\left(\sum_{i=j+1}^{k} \frac{1}{w_{i-1, i}}\right) .
$$

Proof. First we define induced subtrees $T(N)$ of $T$ for $N \in \mathbb{N}$. The vertices of $T(N)$ are those vertices whose distances from $x$ are within $N$. We may apply (11) to get $H(x, y)$ on $T(N)$, which increases as $N$ increases. Then (25) follows easily. We omit the details.

Corollary 4.2. Let $x, y$ be two distinct vertices of a weighted tree $T$. Then $H(x, y)<\infty$ if and only if

$$
\sum_{u \in \mathscr{S}} d_{u}<\infty
$$

where $\mathscr{S}=\{u \in V(T) \mid$ there is a path from $x$ to $u$ not passing through $y\}$.

Corollary 4.3. On the weighted one-dimensional lattice $\mathbb{Z}$,

$$
H(j, j+1)=\frac{\sum_{i \leq j} d_{i}}{w_{j, j+1}} .
$$

Both corollaries follow easily from (25). For unweighted trees, formula (25) was obtained in [Haiyan and Fuji 2004] (see also [Moon 1973]). Formula (26) can be found in [Palacios and Tetali 1996], where it was used to study hitting times for birth and death chains.

Now let $G$ be a locally finite connected weighted graph and $x y \in E(G)$. Then the inequality of Corollary 3.4 still holds: $H(x, y) \leq\left(\operatorname{vol}(G)-d_{y}\right) / w_{x y}$. Next we show that the equality essentially holds when $x y$ is a cut edge of $G$.

Let $\mathscr{S}=\{u \in V(G) \mid$ there is a path from $x$ to $u$ not passing through $y\}$. Let $G^{\prime}$ be the subgraph obtained by removing all vertices in $V(G) /\{\mathscr{Y} \cup y\}$ from $G$. If $x y \in E(G)$ is a cut edge of $G$, note that $H(x, y)$ is the same for random walks on either $G$ or $G^{\prime}$. Moreover, for each spanning tree $T$ of $G^{\prime}$ and $u \in \mathscr{Y}$, there exists a 
path from $x$ to $u$. Therefore,

$$
H(x, y)=\frac{1}{\tau\left(G^{\prime}\right)} \sum_{u \in \mathscr{S}} d_{u} \frac{1}{w_{x y}} \tau\left(G^{\prime}\right)=\frac{1}{w_{x y}} \sum_{u \in \mathscr{S}} d_{u}=\frac{\operatorname{vol}\left(G^{\prime}\right)-d_{y}^{\prime}}{w_{x y}},
$$

where $d_{y}^{\prime}$ is the degree of $y$ in $G^{\prime}$.

Following [Georgakopoulos 2012], we call a weighted graph $G$ reversible if $H(x, y)=H(y, x)$ holds for any $x, y \in V(G)$. For simplicity, we assume that $G$ has no loops, i.e., $w_{x x}=0$ for all $x \in V(G)$, and all edge weights of $G$ are positive. It is interesting to study restrictions on edge weights for a reversible graph $G$.

Conjecture 4.4. Let $G$ be a weighted cycle on $n$ vertices. Assume all edge weights of $G$ are positive. Denote $w_{n, n+1}=w_{n, 1}$.

(i) If $n$ is odd, then $G$ is reversible if and only if there exists some $a>0$ such that $w_{i, i+1}=a$ for all $1 \leq i \leq n$.

(ii) If $n$ is even, then $G$ is reversible if and only if there exist $a, b>0$ such that $w_{1,2}=w_{3,4}=\cdots=w_{n-1, n}=a$ and $w_{2,3}=w_{4,5}=\cdots=w_{n, 1}=b$.

The sufficiency in (ii) follows from Corollary 2.10.

\section{Weight schemes on graphs}

Given a simple, connected, undirected graph $G$ with $n$ vertices, we obtain a weighted graph by assigning a positive number $w_{e}$ to each edge $e \in E(G)$. The hitting and cover times of a simple random walk on $G$ (i.e., $w_{e}=1$, for all $e \in E(G)$ ) have order $O\left(n^{3}\right)$. The work of [Ikeda et al. 2009; Abdullah 2011] showed that if a token knows not only the degree of the current vertex that it is on, but also the degrees of neighboring vertices, we can guarantee $O\left(n^{2}\right)$ hitting times.

In this section, we will denote by $d(u)$ the number of edges adjacent to a vertex $u$ in $G$ and assume that $G$ has no loops.

Lemma 5.1. Let $G$ be connected graph with $n$ vertices and $u_{0}=x, u_{1}, \ldots, u_{l}=y$ a shortest path (achieving minimum $l$ ) connecting any two distinct vertices $x$ and $y$. Then $\sum_{i=0}^{l} d\left(u_{i}\right) \leq 3 n-4$. More precisely,

$$
\sum_{i=0}^{l} d\left(u_{i}\right) \leq \begin{cases}2 n-2 & \text { if } l=1 \\ 3 n-l-3 & \text { if } l \geq 2\end{cases}
$$

Proof. The proof is due to [Ikeda et al. 2009, Theorem 2]. Each vertex of $V(G)$ not lying on the path can be connected to at most 3 vertices of the path, due to its minimality, which also implies that $u_{i}, u_{j}$ are adjacent if and only if $|i-j|=1$. The asserted inequalities follow easily. 
Next we will apply Theorem 3.6 to estimate hitting times under three different weight schemes: $w_{u v}=1 / \sqrt{d(u) d(v)}, 1 / \min \{d(u), d(v)\}$ or $1 / \max \{d(u), d(v)\}$. The leading terms of the bounds in Theorems 5.2 and 5.3 were obtained in [Ikeda et al. 2009, Theorem 2] and [Abdullah 2011, Theorem 68] respectively.

Theorem 5.2. Let $G$ be a graph with assigned weights $w_{u v}=1 / \sqrt{d(u) d(v)}$ for each edge $u v$. Then the hitting time satisfies $H(x, y) \leq 3 n^{2}-9 n+\frac{15}{2}$.

Proof. For the two terms in the right-hand side of (19), we have the estimates

$$
d_{u}=\sum_{v \in \Gamma(u)} \frac{1}{\sqrt{d(u) d(v)}} \leq \frac{1}{2} \sum_{v \in \Gamma(u)}\left(\frac{1}{d(u)}+\frac{1}{d(v)}\right) \leq \frac{1}{2}+\frac{d(u)}{2} \leq \frac{n}{2} .
$$

and, by using $\sum_{u \in V(G)} \sum_{v \in \Gamma(u)} \frac{1}{2}(1 / d(u)+1 / d(v))=n$,

$$
\sum_{\substack{u \in V(G), u \neq y \\ u \notin \Gamma(y)}} d_{u} \leq \sum_{\substack{u \in V(G), u \neq y \\ u \notin \Gamma(y)}} \sum_{v \in \Gamma(u)} \frac{1}{2}\left(\frac{1}{d(u)}+\frac{1}{d(v)}\right) \leq n-1-\frac{d(y)}{2} \leq n-\frac{3}{2} .
$$

Let $u_{0}=x, u_{1}, \ldots, u_{l}=y$ be a shortest path (achieving minimum $l$ ) connecting $x$ and $y$. Then

$$
d(x, y) \leq \sum_{i=0}^{l-1} \sqrt{d\left(u_{i}\right) d\left(u_{i+1}\right)} \leq \sum_{i=0}^{l-1} \frac{d\left(u_{i}\right)+d\left(u_{i+1}\right)}{2} \leq 3 n-5 .
$$

The last inequality follows from Lemma 5.1. By (19), we have

$$
H(x, y) \leq(3 n-5)\left(n-\frac{3}{2}\right)+\frac{1}{2} n=3 n^{2}-9 n+\frac{15}{2},
$$

as claimed.

Theorem 5.3. Let $G$ be a graph with assigned weights $w_{u v}=1 / \min \{d(u), d(v)\}$ for each edge $u v$. Then the hitting time satisfies $H(x, y) \leq 6 n^{2}-18 n+14$.

Proof. For the two terms in the right-hand side of (19), we have

$$
d_{u}=\sum_{v \in \Gamma(u)} \frac{1}{\min \{d(u), d(v)\}} \leq d(u) \leq n-1
$$

and, similarly to (28),

$$
\sum_{\substack{u \in V(G), u \neq y \\ u \notin \Gamma(y)}} d_{u} \leq \sum_{\substack{u \in V(G), u \neq y \\ u \notin \Gamma(y)}} \sum_{v \in \Gamma(u)}\left(\frac{1}{d(u)}+\frac{1}{d(v)}\right) \leq 2 n-3 .
$$


Similarly to (29), we have

$$
d(x, y) \leq \sum_{i=0}^{l-1} \min \left\{d\left(u_{i}\right), d\left(u_{i+1}\right)\right\} \leq \sum_{i=0}^{l-1} d\left(u_{i}\right) \leq 3 n-5 .
$$

The desired upper bound of $H(x, y)$ follows from (19).

Theorem 5.4. Let $G$ be a graph with assigned weights $w_{u v}=1 / \max \{d(u), d(v)\}$ for each edge $u v$. Then the hitting time satisfies $H(x, y) \leq 6 n^{2}-23 n+23$.

Proof. For the two terms in the right-hand side of (19), we have

$$
d_{u}=\sum_{v \in \Gamma(u)} \frac{1}{\max \{d(u), d(v)\}} \leq 1 \quad \text { and } \sum_{\substack{u \in V(G), u \neq y \\ u \notin \Gamma(y)}} d_{u} \leq n-2 .
$$

Similar to (29), we have

$$
d(x, y) \leq \sum_{i=0}^{l-1} \max \left\{d\left(u_{i}\right), d\left(u_{i+1}\right)\right\} \leq \sum_{i=0}^{l-1}\left(d\left(u_{i}\right)+d\left(u_{i+1}\right)-1\right) \leq 6 n-11 .
$$

The desired upper bound of $H(x, y)$ follows from (19).

\section{References}

[Abdullah 2011] M. Abdullah, The cover time of random walks on graphs, Ph.D. thesis, King's College London, 2011. arXiv 1202.5569

[Aldous and Fill 2014] D. Aldous and J. Fill, "Reversible Markov chains and random walks on graphs", Unfinished monograph, 2014, Available at http://www.stat.berkeley.edu/ aldous/RWG/ book.pdf.

[Chandra et al. 1989] A. K. Chandra, P. Raghavan, W. L. Ruzzo, R. Smolensky, and P. Tiwari, "The electrical resistance of a graph captures its commute and cover times", pp. 574-586 in Proc. 21st ACM Symp. Theory of Computing (Seattle, 1989), edited by D. S. Johnson, Association for Computing Machinery, New York, 1989.

[Chang and $\mathrm{Xu} \geq 2015$ ] $\mathrm{X}$. Chang and $\mathrm{H}$. Xu, "Chung-Yau invariants and graphs with symmetric hitting times", preprint, Available at http://pitt.edu/ haoxu/ReversibleGraph.pdf.

[Chung 2011] F. R. K. Chung, "PageRank as a discrete Green's function”, pp. 285-302 in Geometry and analysis, I (Cambridge, MA, 2008), edited by L. Ji, Adv. Lect. Math. (ALM) 17, Int. Press, Somerville, MA, 2011. MR 2012m:68344 Zbl 1255.68109

[Chung and Yau 2000] F. Chung and S.-T. Yau, "Discrete Green's functions", J. Combin. Theory Ser. A 91:1-2 (2000), 191-214. MR 2001g:05073 Zbl 0963.65120

[Georgakopoulos 2012] A. Georgakopoulos, "On walk-regular graphs and graphs with symmetric hitting times", preprint, 2012. arXiv 1211.5689

[Haiyan and Fuji 2004] C. Haiyan and Z. Fuji, "The expected hitting times for graphs with cutpoints", Statist. Probab. Lett. 66:1 (2004), 9-17. MR 2005a:60065 Zbl 1113.60046

[Ikeda et al. 2009] S. Ikeda, I. Kubo, and M. Yamashita, "The hitting and cover times of random walks on finite graphs using local degree information", Theoret. Comput. Sci. 410:1 (2009), 94-100. MR 2010c:60143 Zbl 05509592 
[Lovász 1996] L. Lovász, "Random walks on graphs: A survey", pp. 353-397 in Combinatorics, Paul Erdốs is eighty, II (Keszthely, 1993), edited by D. Miklós et al., Bolyai Soc. Math. Stud. 2, János Bolyai Math. Soc., Budapest, 1996. MR 97a:60097 Zbl 0854.60071

[Moon 1973] J. W. Moon, "Random walks on random trees", J. Austral. Math. Soc. 15 (1973), 42-53. MR 47 \#6525 Zbl 0265.60065

[Palacios and Tetali 1996] J. L. Palacios and P. Tetali, "A note on expected hitting times for birth and death chains", Statist. Probab. Lett. 30:2 (1996), 119-125. MR 97h:60069 Zbl 0883.60081

[Tetali 1991] P. Tetali, "Random walks and the effective resistance of networks", J. Theoret. Probab. 4:1 (1991), 101-109. MR 92c:60097 Zbl 0722.60070

[Xu and Yau 2013a] H. Xu and S.-T. Yau, "Discrete Green's functions and random walks on graphs", J. Combin. Theory Ser. A 120:2 (2013), 483-499. MR 2995053 Zbl 1256.05225

[Xu and Yau 2013b] H. Xu and S. T. Yau, "An explicit formula of hitting times for random walks on graphs", preprint, 2013. To appear in Pure Appl. Math. Q. arXiv 1312.0065

Received February 24, 2014.

\author{
XiAO CHANG \\ DEPARTMENT OF MATHEMATICS \\ UNIVERSITY OF PITTSBURGH \\ 301 THACKERAY HALL \\ PITTSBURGH, PA 15260 \\ UNITED STATES \\ xic58@pitt.edu \\ HAO XU \\ DEPARTMENT OF MATHEMATICS \\ UNIVERSITY OF PITTSBURGH \\ 301 THACKERAY HALL \\ PITTSBURGH, PA 15260 \\ United STATES \\ mathxuhao@gmail.com \\ SHING-TUNG YAU \\ DEPARTMENT OF MATHEMATICS \\ HARVARD UNIVERSITY \\ CAMBridge, MA 02138 \\ UNITED STATES \\ yau@math.harvard.edu
}




\title{
PACIFIC JOURNAL OF MATHEMATICS
}

\author{
msp.org/pjm
}

Founded in 1951 by E. F. Beckenbach (1906-1982) and F. Wolf (1904-1989)

\section{EDITORS}

Don Blasius (Managing Editor)

Department of Mathematics

University of California

Los Angeles, CA 90095-1555

blasius@math.ucla.edu

\author{
Paul Balmer \\ Department of Mathematics \\ University of California \\ Los Angeles, CA 90095-1555 \\ balmer@math.ucla.edu \\ Robert Finn \\ Department of Mathematics \\ Stanford University \\ Stanford, CA 94305-2125 \\ finn@math.stanford.edu \\ Sorin Popa \\ Department of Mathematics \\ University of California \\ Los Angeles, CA 90095-1555 \\ popa@math.ucla.edu
}

\author{
Vyjayanthi Chari \\ Department of Mathematics \\ University of California \\ Riverside, CA 92521-0135 \\ chari@math.ucr.edu \\ Kefeng Liu \\ Department of Mathematics \\ University of California \\ Los Angeles, CA 90095-1555 \\ liu@math.ucla.edu \\ Jie Qing \\ Department of Mathematics \\ University of California \\ Santa Cruz, CA 95064 \\ qing@ cats.ucsc.edu
}

\section{PRODUCTION}

Silvio Levy, Scientific Editor, production@msp.org

\section{SUPPORTING INSTITUTIONS}

ACADEMIA SINICA, TAIPEI

CALIFORNIA INST. OF TECHNOLOGY

INST. DE MATEMÁTICA PURA E APLICADA

KEIO UNIVERSITY

MATH. SCIENCES RESEARCH INSTITUTE

NEW MEXICO STATE UNIV.

OREGON STATE UNIV.

\author{
STANFORD UNIVERSITY \\ UNIV. OF BRITISH COLUMBIA \\ UNIV. OF CALIFORNIA, BERKELEY \\ UNIV. OF CALIFORNIA, DAVIS \\ UNIV. OF CALIFORNIA, LOS ANGELES \\ UNIV. OF CALIFORNIA, RIVERSIDE \\ UNIV. OF CALIFORNIA, SAN DIEGO \\ UNIV. OF CALIF., SANTA BARBARA
}

\author{
Daryl Cooper \\ Department of Mathematics \\ University of California \\ Santa Barbara, CA 93106-3080 \\ cooper@math.ucsb.edu \\ Jiang-Hua Lu \\ Department of Mathematics \\ The University of Hong Kong \\ Pokfulam Rd., Hong Kong \\ jhlu@maths.hku.hk \\ Paul Yang \\ Department of Mathematics \\ Princeton University \\ Princeton NJ 08544-1000 \\ yang@math.princeton.edu
}

These supporting institutions contribute to the cost of publication of this Journal, but they are not owners or publishers and have no responsibility for its contents or policies.

See inside back cover or msp.org/pjm for submission instructions.

The subscription price for 2015 is US \$420/year for the electronic version, and \$570/year for print and electronic.

Subscriptions, requests for back issues and changes of subscribers address should be sent to Pacific Journal of Mathematics, P.O. Box 4163, Berkeley, CA 94704-0163, U.S.A. The Pacific Journal of Mathematics is indexed by Mathematical Reviews, Zentralblatt MATH, PASCAL CNRS Index, Referativnyi Zhurnal, Current Mathematical Publications and Web of Knowledge (Science Citation Index).

The Pacific Journal of Mathematics (ISSN 0030-8730) at the University of California, c/o Department of Mathematics, 798 Evans Hall \#3840, Berkeley, CA 94720-3840, is published twelve times a year. Periodical rate postage paid at Berkeley, CA 94704, and additional mailing offices. POSTMASTER: send address changes to Pacific Journal of Mathematics, P.O. Box 4163, Berkeley, CA 94704-0163.

PJM peer review and production are managed by EditFLOW ${ }^{\circledR}$ from Mathematical Sciences Publishers.

\section{PUBLISHED BY}

\section{mathematical sciences publishers \\ nonprofit scientific publishing}

http://msp.org/

(C) 2015 Mathematical Sciences Publishers 


\section{PACIFIC JOURNAL OF MATHEMATICS}

Volume $273 \quad$ No. $1 \quad$ January 2015

Maximal estimates for Schrödinger equations with inverse-square potential 1

ChangXing MiaO, JUNYong Zhang and JiQiang Zheng

Vassiliev Invariants of Virtual Legendrian Knots

PATRICIA CAHN and ASA LEVI

Some results on the generic vanishing of Koszul cohomology via

deformation theory

JIE WANG

Conformal metrics with constant curvature one and finitely many conical

singularities on compact Riemann surfaces

QING Chen, WeI WANG, Yingyi Wu and BIN XU

$\mathbb{Q}$-bases of the Néron-Severi groups of certain elliptic surfaces

MASAMICHI KURODA

On a prime zeta function of a graph

TAKehiro Hasegawa and SEIKEN SAIto

On Whittaker modules for a Lie algebra arising from the 2-dimensional torus 147

SHaObin TAN, QING WANG and ChENGKANG XU

Fréchet quantum supergroups

AXEL DE GOURSAC

Generators of the Gauss-Picard modular group in three complex dimensions

BaoHua Xie, JieYan Wang and YuePing Jiang

Complete characterization of isolated homogeneous hypersurface singularities

STEPHEN YAu and HuAiQING ZuO

A theorem of Mœglin and Waldspurger for covering groups

SHIV PRAKASH PATEL

Spanning trees and random walks on weighted graphs 